\section{(1) \\ CrossMark}

\title{
Is mitochondrial dysfunction a driving mechanism linking COPD to nonsmall cell lung carcinoma?
}

\author{
Francois Ng Kee Kwong ${ }^{1,2}$, Andrew G. Nicholson ${ }^{1,2}$, Celeste L. Harrison ${ }^{3}$, \\ Philip M. Hansbro ${ }^{3}$, Ian M. Adcock ${ }^{4}$ and Kian Fan Chung ${ }^{1}$
}

Affiliations: ${ }^{1}$ Experimental Studies, National Heart and Lung Institute, Imperial College London, London, UK. ${ }^{2}$ Dept of Histopathology, Royal Brompton and Harefield NHS Foundation Trust, London, UK. ${ }^{3}$ School of Biomedical Sciences and Pharmacy, Newcastle, Australia. ${ }^{4}$ Airways Disease, National Heart and Lung Institute, Imperial College London, London, UK.

Correspondence: Francois Ng Kee Kwong, National Heart and Lung Institute, Imperial College, Cale Street, London, SW3 6LY, UK. E-mail: f.ng-kee-kwongdimperial.ac.uk

@ERSpublications

We postulate that patients with COPD have increased risk of lung cancer as a result of mitochondrial dysfunction http://ow.ly/qzGv30fiAUm

Cite this article as: Ng Kee Kwong F, Nicholson AG, Harrison CL, et al. Is mitochondrial dysfunction a driving mechanism linking COPD to nonsmall cell lung carcinoma? Eur Respir Rev 2017; 26: 170040 [https://doi.org/10.1183/16000617.0040-2017].

ABSTRACT Chronic obstructive pulmonary disease (COPD) patients are at increased risk of developing nonsmall cell lung carcinoma, irrespective of their smoking history. Although the mechanisms behind this observation are not clear, established drivers of carcinogenesis in COPD include oxidative stress and sustained chronic inflammation. Mitochondria are critical in these two processes and recent evidence links increased oxidative stress in COPD patients to mitochondrial damage. We therefore postulate that mitochondrial damage in COPD patients leads to increased oxidative stress and chronic inflammation, thereby increasing the risk of carcinogenesis.

The functional state of the mitochondrion is dependent on the balance between its biogenesis and degradation (mitophagy). Dysfunctional mitochondria are a source of oxidative stress and inflammasome activation. In COPD, there is impaired translocation of the ubiquitin-related degradation molecule Parkin following activation of the Pink1 mitophagy pathway, resulting in excessive dysfunctional mitochondria. We hypothesise that deranged pathways in mitochondrial biogenesis and mitophagy in COPD can account for the increased risk in carcinogenesis. To test this hypothesis, animal models exposed to cigarette smoke and developing emphysema and lung cancer should be developed. In the future, the use of mitochondriabased antioxidants should be studied as an adjunct with the aim of reducing the risk of COPD-associated cancer.

Received: April 022017 | Accepted after revision: July 172017

Support statement: I.M. Adcock is supported by the Wellcome Trust (093080/Z/10/Z) and by the Dunhill Medical Trust (R368/0714). I.M. Adcock and K.F. Chung are supported by the Medical Research Council/Association of the British Pharmaceutical Industry (MRC/ABPI) COPDMAP consortium (G1001367/1). P.M. Hansbro is supported by grants from the Cancer Council NSW, the Australian Lung Foundation and the University of Newcastle (Australia), a National Health and Medical Research Council (Australia) Principal Research Fellowship and a Brawn Fellowship from the Faculty of Health and Medicine, University of Newcastle (Australia).

Conflict of interest: None declared.

Provenance: Submitted article, peer reviewed.

Copyright CERS 2017. ERR articles are open access and distributed under the terms of the Creative Commons Attribution Non-Commercial Licence 4.0. 


\section{Background}

Lung cancer is a common and deadly malignancy worldwide [1]. Nonsmall cell lung carcinoma (NSCLC), which accounts for $87 \%$ of lung cancer, usually presents at a late stage with locally advanced or metastatic disease in $80 \%$ of cases [2], which largely explains its dismal prognosis of $15 \%$ survival at 5 years post-diagnosis [3]. The advent of molecular targeted therapies has led to an improved outcome in patients with metastatic pulmonary adenocarcinoma that harbours an activated oncogene, such as epidermal growth factor receptor (EGFR) [4, 5]. However, these patients constitute only a fraction of the total number of lung cancer sufferers, and even in these patients long-term disease control is not achieved [6]. Novel detection and treatment strategies for lung cancer are therefore urgently required to improve this dismal current prognosis of lung cancer. To achieve this, we need to understand how known risk factors can trigger the mechanisms of lung carcinogenesis in its early stages.

\section{The clinical links between COPD and lung cancer}

Up to $85 \%$ of lung cancer is related to smoking. Yet, only $15 \%$ of smokers will develop lung cancer and $10-25 \%$ of lung cancer sufferers are never-smokers [7]. Of the lung cancer smokers, between $50 \%$ and $70 \%$ have pre-existing chronic obstructive pulmonary disease (COPD) prior to cancer diagnosis $[8,9]$ The annual incidence of lung cancer arising from patients with COPD has been reported to be $0.8-1.7 \%[10,11]$.

COPD per se is a risk factor for developing lung cancer, irrespective of the fact that smoking is a common risk factor to both conditions. As early as 1986, an association between airflow obstruction and increased incidence of lung cancer was noted [12]. This observation has since been confirmed in larger studies that have carefully controlled for cigarette smoke consumption [8, 10, 13-16]. Smokers with airflow obstruction have a five-fold increased risk of lung cancer compared with those with normal lung function [8]. A reduction of $10 \%$ in the predicted forced expiratory volume in $1 \mathrm{~s}$ was associated with a nearly three-fold greater lung cancer risk [16]. In a large cohort of $>2500$ COPD patients followed up to 90 months, 215 patients developed lung cancer (incidence density of 16.7 per 1000 person-years) [17]. In addition, stage 1 NSCLC patients, who undergo lung resection, are more likely to have tumour recurrence if they have COPD [18].

As well as airflow obstruction, emphysema diagnosed by computed tomography (CT) is also an independent risk factor for lung cancer $[19,20]$. A meta-analysis also concluded that CT-diagnosed emphysema was a negative prognostic factor for the overall survival of patients, independent of tumour staging [21]. A recent study from the Multi-Ethnic Study of Atherosclerosis group showed that emphysema on CT in the general population, irrespective of their smoking history, conferred a three-fold increased risk of respiratory mortality caused by lung cancer [22]. This effect on outcome was also dependent on the severity of emphysema in two additional studies [23, 24]. Lung cancers tend to arise within areas of worse regional emphysema, as detected on CT [23, 24].

The above observations suggest that the risk of developing lung cancer in COPD patients is not solely related to exposure to smoking, but that the development of airflow obstruction and emphysema are likely to be additional drivers of lung carcinogenesis.

\section{Molecular drivers of COPD-associated lung cancer in smokers and nonsmokers}

Lung cancer patients who have smoked differ markedly in age of presentation, sex and geographical incidence, compared with those who have not. The histological profile and molecular drivers of carcinogenesis are also dependent on the patients' smoking status, although there is some overlap between the two groups of patients.

\section{Nonsmokers}

Nonsmokers who develop lung cancer tend to be younger, are more likely to be female and are more common among Asians [25]. They also have a genetic predisposition to alterations in CYP1A1 and GSTM1 genes (carcinogen metabolism), XRCC1 and ERCC2 genes (DNA repair genes) and the IL10 gene (inflammatory response) [25, 26].

Adenocarcinomas form a much higher proportion of the histological classification of NSCLC in nonsmokers (70\%) than in current (40\%) and former smokers (47\%). Histologically, peripheral adenocarcinomas in nonsmokers are preceded by atypical adenomatous hyperplasia and adenocarcinoma in situ and are considered to originate from the terminal respiratory unit [27], which consists of non-ciliated small bronchioles and pneumocytes [27].

Lung cancer in nonsmokers is more likely to be caused by a single driver oncogene mutation, unlike in smokers [28]. Over half of primary lung adenocarcinomas harbour a known driver mutation, most commonly in KRAS or EGFR [26]. In addition, EGFR mutations are more common in nonsmokers, being 
present in up to $40 \%$ of adenocarcinomas from an Asian nonsmoking population [29]. Other less commonly altered genes in nonsmokers are ALK and ROS1, which account for 7-10\% of NSCLCs. These mutations are rare in squamous cell carcinoma (SQCC), which constitute only $18 \%$ of NSCLC in nonsmokers [29].

\section{Smokers}

Lung cancer in smokers tends to be central in location with a predominance of SQCC [30]. It is thought that the effect of carcinogens from tobacco smoke is greater in the proximal than the distal airways. When both central and peripheral tumours are taken into account in smokers, the histological classification consists of $48 \%$ of SQCC and $44 \%$ of adenocarcinoma [31].

KRAS mutations are more common in adenocarcinomas from smokers than those from nonsmokers [26, 32]. However, frequent and clinically relevant driver mutations in adenocarcinoma (in EGFR and KRAS) are present in $<5 \%$ of SQCC patients [29]. The most common genomic alterations in SQCC are the loss of p53 and CDKN2A, but smoking-associated cancers harbour more widespread genetic changes. There are currently no clinically approved target agents for SQCC being used for treatment. There is therefore an urgent need to find novel ways of treating NSCLC associated with smoking, whether they are adenocarcinomas or SQCCs.

COPD-associated NSCLC exhibited a low prevalence of EGFR and ALK driver mutations compared with NSCLC patients who did not have COPD [33] and is not correlated with KRAS mutations [34]. However, in these two studies $[33,34]$, SQCC and adenocarcinoma cases were not studied separately and were grouped together as NSCLC patients. Future studies investigating the effect of COPD status on the presence of driver mutations will need to separate these patients, according to the histology of their cancers and their smoking status.

There is debate about whether the COPD status of lung cancer patients is a risk factor for a particular histological subtype of NSCLC (adenocarcinoma versus SQCC). PAPI et al. [35] were the first to suggest that COPD was an independent risk factor for SQCC, but not adenocarcinoma. This observation seemed supported by a later study investigating the lung cancer subtypes associated with emphysema on CT [21]. However, a large cohort of 565 lung cancer cases from the Mayo clinic showed that emphysema is a risk factor for both adenocarcinoma and SQCC [36].

Overall, COPD-associated NSCLC consists of a mixture of histological subtypes of NSCLC in both central and peripheral locations. In a cohort of 230 patients with airflow limitation and emphysema, SHIN et al. [37] found $37 \%$ of patients with central lung cancer, of which $76 \%$ were SQCC, $17 \%$ small cell carcinoma and $5 \%$ adenocarcinomas. In the peripheral lung cancer, the histological subtype of NSCLC was dependent on whether the tumour arose within an area of emphysema. SQCC was more frequently found in emphysema areas than areas without emphysema, while the reverse was true for adenocarcinoma [37].

SQCCs are derived from bronchial epithelial cells with the well-established pathogenic sequence of squamous metaplasia, dyplasia and carcinoma in situ in the central airways [30]. The SQCC may also undergo the same pathological sequence in the smaller airways, but peripheral SQCCs are less common as it is thought that the magnitude of the toxic effects of smoke are stronger in the central airways.

There is more debate on the cells of origin of adenocarcinomas. Immunohistochemical studies of human lung cancer suggest that they derive from the terminal respiratory unit [27, 30]. Animal models of lung adenocarcinoma also suggest that they tend to arise mostly from the terminal respiratory unit, in particular the type 2 alveolar cells, but may also arise from a more proximal bronchioalveolar stem cells niche [38].

Despite the low incidence of the aforementioned oncogene driver mutations, there is some evidence that COPD-associated NSCLC may be driven by specific genetic mutations. In a limited direct comparison of SQCC from patients with or without COPD, Boelens et al. [39] showed that $>370$ genes are differentially expressed, with 34 genes located on chromosome $5 \mathrm{q}$ and 44 genes related to mitochondrial function. Aberrant DNA methylation of promoter sequences is a recognised factor in the pathogenesis of human cancer. The differentially methylated and expressed genes in COPD-associated NSCLC versus non-COPD-associated NSCLC are mainly involved in the immune response, in particular the innate defence response, dendritic cell and lymphocyte trafficking [40], which is associated with a reduced infiltration of CD3- and CD4-positive T-cells in the tumour microenvironment. The methylation of tumour suppressor genes and apoptosis-related genes, such as mitochondrial transcription factor A, in COPD-associated NSCLC has also been demonstrated [41, 42]. A recent study by XIAO et al. [43] showed that lung adenocarcinoma patients with COPD have a higher prevalence of LDL receptor protein $1 \mathrm{~B}$ mutations than those without COPD. 
Oxidative stress and inflammation as the link between COPD and carcinogenesis Multiple mechanisms, such as oxidative stress, chronic inflammation and genetic factors, have been proposed to account for the link between COPD and lung cancer and have been reviewed elsewhere [4446]. Of those, oxidative stress and sustained chronic inflammation are well-established drivers of carcinogenesis [47, 48]. Mitochondrial dysfunction is central to these two drivers of carcinogenesis in COPD patients.

Smoking is a source of toxic chemicals and provides excessive exposure of the airways to reactive oxygen species (ROS). However, COPD patients have an increase in oxidative stress compared with non-COPD smokers. The airways of patients with COPD demonstrate an increase in mitochondrial ROS compared with healthy smokers and nonsmokers [49], an effect at least partly due to mitochondrial damage. Pathways involved in oxidant/antioxidant responses, such as superoxide dismutase, were among the most differentially expressed gene pathways in the airways of COPD patients [50]. COPD patients also have an impairment of antioxidant defences, such as NRF2 (nuclear factor erythroid 2-related factor 2) [51].

Excess ROS cause direct damage to genomic DNA, proteins and lipids [52]. DNA damage leads to point mutations, strand breaks and cross-linking that may result in somatic mutations, which accumulate with age and increases the predisposition to cancer. As expected, oxidative DNA damage is prominent in COPD lungs [53, 54]. The expression of DNA damage/repair proteins also increases significantly with worsening severity of the pre-neoplastic changes in the lung [55], further strengthening the hypothesis that oxidative DNA damage is important in lung carcinogenesis. Since NSCLC originates from bronchial epithelial cells or the terminal respiratory unit, it was thought that COPD-related molecular differences in the epithelium would be magnified in NSCLC because of clonal expansion [56]. Indeed, oxidative stress-related proteins, such as catalase and thioredoxin, in bronchoalveolar lavage fluid are differentially expressed in both COPD and COPD-associated NSCLC compared with nonsmokers [57].

The effect of increased oxidative stress on carcinogenesis may also be mediated by the induction of cellular senescence. Emphysematous lungs show evidence of cellular senescence and accelerated ageing with shortened telomeres and decreased anti-ageing molecules [58]. As well as increased oxidative stress, cell senescence is also known to be induced by mitochondrial dysfunction [59-62]. In addition to showing irreversible growth arrest, senescent cells show a senescence-associated secretory phenotype, containing factors with pro-tumorigenic properties $[63,64]$. Inflammation is another critical driver for both COPD and lung cancer. Macrophages and neutrophils infiltrate the lungs of all smokers. However, patients with COPD develop more pronounced inflammation than smokers without COPD [54]. This inflammatory infiltrate correlates positively with disease severity. This is further compounded by the impaired immune response in COPD patients [65]. For example, programmed cell death protein (PD)-1 induces a loss of cytotoxic function in T-cells and is upregulated in T-cells derived from patients with COPD [66].

Up to $20 \%$ of cancers are related to chronic inflammation caused by infection, irritants or autoimmunity [67]. Large epidemiological studies have provided indirect evidence that chronic inflammation is associated with lung cancer. In a cohort of $>7000$ patients without known malignancy followed for $\sim 10$ years, the likelihood of developing cancer was shown to be increased if C-reactive protein was $>3 \mathrm{mg} \cdot \mathrm{dL}^{-1}[68]$. A retrospective study of $>10000$ patients with COPD found that the risk of lung cancer was decreased among patients taking inhaled corticosteroids at a dose of $>1200 \mu \mathrm{g} \cdot \mathrm{day}^{-1}$ compared with patients not taking inhaled corticosteroids or taking lower doses [69]. Therefore, persistent inflammation is associated with an increased risk of malignancy. This may be partially related to the activation of hypoxia-inducible factor (HIF)-1 $\alpha$ via hypoxia-controlled mechanisms in inflammation or the release of the inflammasome via the mitochondrion, as will be discussed later in this review.

Although there is increased inflammation at the initiation stage of COPD-associated carcinoma, there is as yet no known evidence whether there is increased inflammation with established COPD-associated cancer. Further studies are awaited to determine whether COPD-associated NSCLCs have an inflammatory stroma.

\section{Mitochondrial dysfunction in lung cancer}

Under physiological conditions, the mitochondrion is the main source of ROS, as a by-product of ATP production from the oxidative phosphorylation system [70]. The mitochondrion is also the hub for sensing inflammatory damage signals and is the site of the initiation of the inflammatory response. In particular, mitochondria-derived proteins, such as mitochondrial antiviral signalling protein (MAVS), initiate the NLRP3 inflammasome [71]. Inflammasomes are multimolecular complexes composed of a sensor protein, an adaptor protein ASC and caspase-1. Conformational changes of this multimolecular complex lead to the activation of caspase- 1 and hence the induction of the pro-inflammatory cytokines, such as interleukin (IL)-1 $\beta$ and IL-18 [72]. Currently, the identified inflammasome sensor proteins include 
the nucleotide-binding domain and leucine-rich repeat containing proteins (NLR), such as NLRP1, $-3,-4$ and -6 and NAIP5, as well as the DNA-sensing complex of the absent in melanoma 2 (AIM2) protein [73].

Deranged mitochondrial function in cancer was suspected as far back as 1956, when WARBURG [74] discovered that cancer cells preferentially produce ATP by glycolysis, rather than by oxidative phosphorylation as in normal cells, even in the presence of oxygen. This metabolic adaptation and enhanced glucose uptake provides cancer cells with glucose metabolites, raw materials in the biosynthesis of nucleic acids, amino acids and lipids in these rapidly dividing cell populations. These observations led WARBURG [74] to postulate that mitochondrial respiration defects are the basis for carcinogenesis. Although only a handful of cancers are now known to result from mutations in the metabolic enzymes within the mitochondrion, somatic mutations of the mitochondrial genome are established factors in carcinogenesis [75].

However, there remains a paucity of studies detailing the mitochondrial abnormalities present in lung cancer, when compared with other cancer types. Mitochondrial mutations have been reported in human lung cancer, with some evidence that they are linked to the survival of the patients [76-80]. Damage to lung mitochondrial DNA and related dysfunction has been linked with cigarette smoking [81-83]. Furthermore, this has been associated with the accumulation of mitochondrial DNA adducts resulting from tobacco-related carcinogen metabolism over time, as a result of insufficient repair processes in rats [84, 85]. Certainly, DNA adducts formed as a result of tobacco-specific nitrosamine 4-(methylnitrosamino)-1-(3-pyridyl)-1-butanone metabolism induced both nuclear and mitochondrial DNA damage in exposed Caenorhabditis elegans, which was associated with reduced ATP production and oxygen consumption [86]. At present, investigations in the context of mouse models of human lung cancer are limited, but may provide a critical understanding of the underlying mechanisms of lung tumorigenesis.

It is postulated that mitochondrial dysfunction is involved in the initiation of both centrally and peripherally located COPD-associated NSCLC. Impaired mitochondrial function in COPD contributes to the production of excess ROS from cigarette smoke exposure within both central and peripheral airways, as well as the terminal respiratory unit. However, there is a different progenitor cell population lining the airways at different anatomical sites, leading to different histological subtypes of cancer (SQCC centrally and a mixture adenocarcinoma and SQCC peripherally).

\section{Altered mitochondrial dynamics in COPD and NSCLC}

Cigarette smoke extract induces alterations in mitochondrial structure and function and leads to increased expression of the mitochondrial fusion protein, Mfn-2, in alveolar epithelial cells [87]. However, there are as yet no studies suggesting that alterations in mitochondrial fusion or fission are involved in the pathogenesis of COPD. There is conflicting evidence about the role of these processes in NSCLC. Evidence of mitochondrial fission in human lung cancer cell lines and adenocarcinoma has been reported, with an increase in dynamin-related protein (DRP)-1 and decrease in Mfn-2 compared with background lung tissue [88]. When this imbalance was corrected by increasing Mfn-2 expression and decreasing DRP-1 in cell lines, there was a reduction of cell proliferation and an increase in apoptosis [88]. However, Lou et al. [89] demonstrated that, in human lung adenocarcinoma, the expression of Mfn-2 was higher than in adjacent background lung tissue.

Mitochondrial function is also intimately linked with oxygen homeostasis. Hypoxia, a defining feature of COPD and lung cancer, elicits a number of adaptive responses via HIF-1 $\alpha$. The latter is expressed in the large airways of patients with COPD, but not of smokers without COPD or of nonsmokers [90]. HIF-1 $\alpha$ is also overexpressed in $32-56 \%$ of NSCLC and is associated with worse prognosis [91, 92].

HIF- $1 \alpha$ promotes glycolytic energy production by inducing genes that encode glucose transporters and glycolytic enzymes and reduces oxidative phosphorylation within mitochondria [93]. This is the metabolic phenotype described by WARBURG [74] in cancer cells. COPD patients also produce glucose at a faster rate than healthy control subjects, with a higher glucose clearance [93]. HIF-1 $\alpha$ may therefore provide the link between hypoxia and mitochondria-based metabolic changes in both COPD and lung cancer.

\section{Mitophagy in COPD and lung cancer}

Mitophagy is initiated by membrane depolarisation or hypoxia, and is triggered by three main molecular pathways: NIP3-like protein X (NIX)/BNIP3L, Pink1/Parkin and Fun14 domain containing 1 (FUNDC1) [94-98]. BNIP3L and NIX initiate mitophagy through interactions with LC3 (microtubule associated protein 1 light chain 3 alpha)-related molecules [99, 100], thereby linking the mitochondria directly to the autophagosome for degradation. Pink1 is a serine/threonine kinase that phosphorylates the fusion protein Mfn-2 [101, 102], which acts as a receptor for the E3 ubiquitin ligase Parkin [90]. Hypoxia promotes mitophagy through dephosphorylation of the FUNDC1 protein, thereby facilitating its interaction with LC3 [98]. 
Depending on the cellular context, mitophagy has a positive role in controlling oxidative stress and inflammation. Damaged mitochondria are prone to uncoupling and therefore lead to the release of further ROS [103]. The activation of mitophagy therefore leads to the removal of damaged mitochondria and reduces the release of ROS. Impaired mitophagy reduces clearance of damaged mitochondria, resulting in increased ROS production and mitochondrial DNA accumulation in the cytoplasm. The subsequent release of mitochondrial ROS leads to inflammation, particularly the enhanced expression of IL-1 $\beta$ and IL-18 [104-106]. Mitophagy is therefore also a negative regulator of inflammasome activation [106, 107]. Therefore, mitophagy can be considered as having a tumour suppressive function by limiting the production of excessive ROS [108] and by inhibiting inflammasome activation.

There is dysregulation of the Pink1/Parkin mitophagy pathway in the pathogenesis of COPD. MizumurA et al. [109] showed there was stabilisation of Pink1 in cultured pulmonary epithelial cells exposed to cigarette smoke. Somewhat unexpectedly therefore, a later study showed that there was decreased expression of Parkin in COPD lungs [110]. One potential explanation is the observation that cigarette smoke impairs the translocation of Parkin to damaged mitochondria and leads to the accumulation of damaged mitochondria in lung fibroblasts and small airway epithelial cells [111]. Therefore, in COPD, there is activation of Pink1, but impaired translocation of Parkin to the mitochondria. The decrease in Parkin within COPD lungs was associated with increased ROS and senescence of the bronchial epithelial cells [110].

Dysregulation of the Pink1/Parkin pathway has also been demonstrated in lung cancer. Diffuse cytoplasmic expression of Pink1 was observed in lung SQCC, which contrasted with the granular cytoplasmic pattern found in normal lung tissue [112]. The lack of a granular cytoplasmic pattern, consistent with mitochondrial expression, suggests that aberrant expression of Pink1 may be associated with lung carcinogenesis [112]. Human lung adenocarcinomas also express different Parkin isoforms that may be linked to regulation of apoptosis, as well as mitophagy and mitochondrial fusion [113].

We therefore speculate that the dysfunctional Pink1/Parkin mitophagy pathway in COPD leads to an excess of degenerate mitochondria and subsequent enhanced ROS release (figure 1). This would contribute to oxidative DNA damage and increased chronic inflammation, leading to an increased likelihood for carcinogenesis. Whether the other pathways of mitophagy, activated by BNIP3 or FUNDC1, are affected in COPD and lung cancer is not currently known.

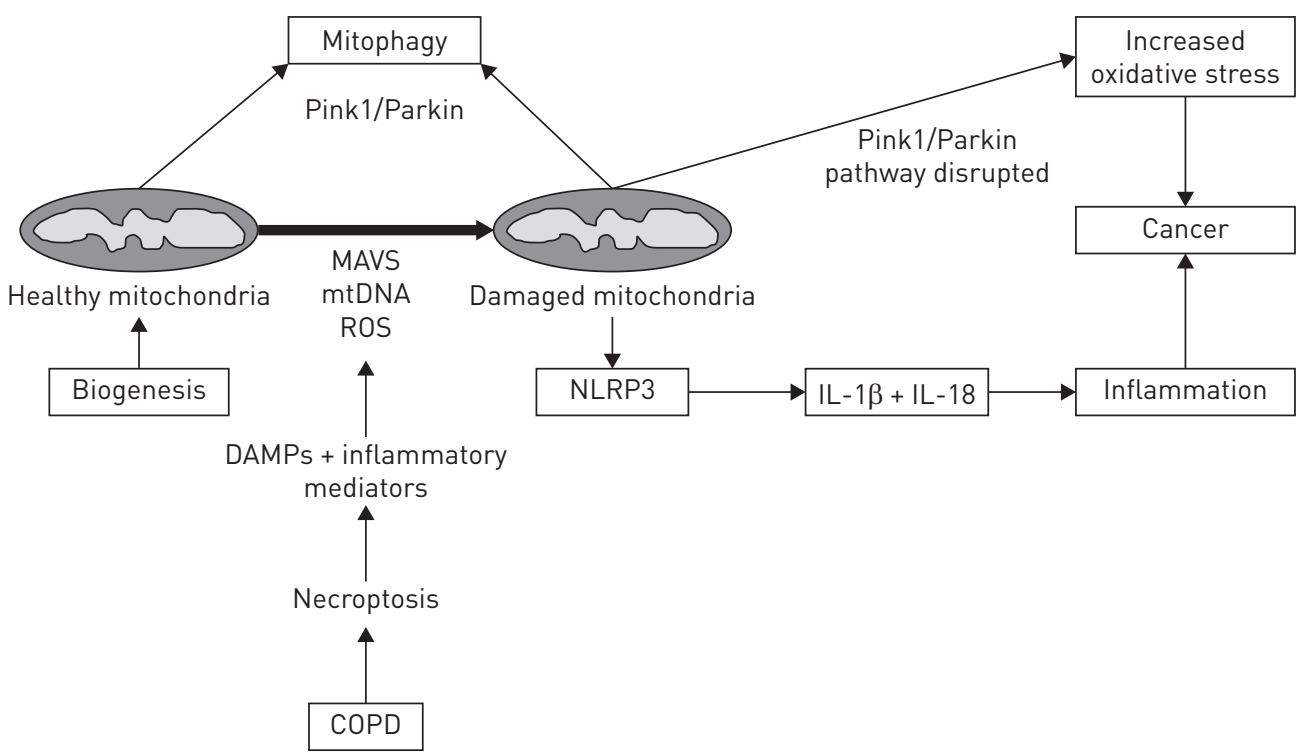

FIGURE 1 The mechanisms by which mitochondrial dysfunction in chronic obstructive pulmonary disease (COPD) lead to increased oxidative stress and inflammation, drivers of carcinogenesis. In COPD, there is an increase in mitochondrial damage as a result of the release of mitochondrial antiviral signalling protein (MAVS), reactive oxygen species (ROS) and mitochondrial DNA (mtDNA). Damaged mitochondria should undergo physiological degradation, mitophagy. However, in COPD, the Pink1/Parkin mitophagy pathway is disrupted and therefore mitophagy is impaired. The persisting damaged mitochondria are a source of oxidants and can induce the NLRP3 inflammasome pathway. IL: interleukin; DAMPs: damage-associated molecular patterns. 


\section{Inflammasomes in COPD and lung cancer}

In COPD, there is upregulation of the ATP-NLRP3 inflammasome pathway [114], with the release of the downstream molecules, IL-1 $\beta$ and IL-18 $[115,116]$. This is partially dependent on cigarette smoke activation of a critical adaptor molecule of the NLRP3 pathway, MAVS [117]. Inhibition of MAVS and NLRP3 inflammasome pathway could therefore reduce the chronic inflammation associated with COPD.

Components of the inflammasome pathway are also overexpressed in lung cancer, with AIM2 and NLRP3 as the major scaffolding proteins [118]. AIM2 is overexpressed in all subtypes of NSCLC while NLRP3 is upregulated in adenocarcinoma, with expression limited to the malignant epithelial cells [118]. The role of the inflammasome activation in the pathogenesis of lung cancer remains uncertain. However, activation of the NLRP3 inflammasome increases the proliferation and migration of lung cancer cell lines [119]. It has also been suggested that inflammasome activation could contribute to the immune response of lung cancer [118], but there is no experimental evidence to support this hypothesis currently. Further studies should investigate whether the targeting of the inflammasome activation in established lung cancers would alter the associated immune response and hence improve outcome.

\section{Targeting mitochondria to reduce COPD-associated carcinogenesis}

In other oxidative stress-related pathologies, mitochondria-targeted antioxidants have been used in clinical trials [120]. Further studies of the role of mitochondrial dysfunction in COPD-associated NSCLC hold the promise that mitochondria-based drugs can be used as an adjunct for cancer treatment or as a prophylactic drug in reducing lung cancer incidence in patients with COPD. For example, functional studies, using mitochondria-targeted antioxidants, are required to determine the feasibility of reducing the incidence of lung cancer in COPD by reducing oxidative stress. This will require the development of animal models which combine both emphysema and lung cancer (as discussed in the next section).

The best characterised mitochondria-targeted antioxidant to date is MitoQ, which is made of lipophilic triphenylphosphonium cations, attached to coenzyme Q10, allowing it to easily pass through phospholipid bilayers. MitoQ is driven by the large mitochondrial membrane potential and accumulates at a high concentration in the mitochondria [120]. It is then reduced by the respiratory chain to its active ubiquinol form, which prevents lipid peroxidation and mitochondrial damage. In clinical trials, MitoQ has been used safely in patients with Alzheimer's disease and reduced liver damage following hepatitis C infection [120].

A different class of mitochondria-targeted antioxidants are small cell-permeable peptides, called SS (SzetoSchiller) peptides [121], that contain an amino acid sequence that allows them to freely penetrate cells in an energy-independent nonsaturable manner and partition in the inner mitochondrial membrane. They scavenge ROS and inhibit low-density lipid peroxidation.

As well as the treatment of lung cancer, mitochondrial dysfunction may be further explored to reduce the incidence of lung cancer. Currently, it is not possible to screen the entire at-risk population for lung cancer because of the large numbers of individuals concerned. Screening a more limited population may be feasible. Therefore, further criteria are required to restrict screening to patients who are at even greater risk than the average smoker. Patients with COPD define a population that is a particularly high risk for lung cancer and may represent a target for screening. The expression of some mitochondria-related proteins is related to the outcome of lung cancer $[122,123]$, thereby raising the possibility that they can be used as biomarkers for lung cancer.

\section{Mouse models of COPD-associated lung cancer}

Murine models that accurately mimic the transformation of COPD to NSCLC are urgently required. Murine models of lung cancer are widely available [124], but a large proportion comprises transgenic mice with deletions of tumour-suppressor genes or with lung-specific overexpression of key oncogenes [125, 126] or patient-derived xenograft models [127]. These models are excellent for investigating specific signalling pathway interactions involved in tumorigenesis, but they do not model the effects of cigarette smoking and COPD on lung carcinogenesis.

In order to incorporate the risk factors for lung cancer, and best represent human disease, carcinogen models need to be employed in conjunction with cigarette smoke exposure. Importantly, susceptibility to developing lung cancer either spontaneously or as a result of chemical induction/smoke exposure varies greatly between wild-type mouse strains much in the way it does in humans. The C57BL/6 mouse strain is regarded as resistant, whereas BALB/c mice exhibit an intermediate susceptibility [128, 129]. The strains displaying the highest level of susceptibility are A/J and SWR mice, and these strains are most commonly used to model lung tumours in vivo. Although these models are useful, they are very long and expensive to run, meaning that critical information that may lead to improvements for lung cancer patients is generated slowly. 
Thus, the development of short-term models that develop the hallmarks of cigarette smoke-induced COPD and lung cancer is vitally important to gain a better understanding of the mechanisms of lung cancer in a timely manner. We have recently refined this model of combined carcinogen and cigarette smoke exposure down to 17 weeks based on our more rapid model of induction of COPD (unpublished data).

\section{Conclusion}

Novel strategies in the prevention and treatment of lung cancer are required. We need to continue exploring new pathways by which lung carcinogenesis is initiated to provide further targeted treatment regimes for specific subsets of lung cancer, one of which may be mitochondrial dysfunction-driven COPD-associated NSCLC.

Established drivers of carcinogenesis in COPD include oxidative stress and sustained chronic inflammation. Dysfunctional mitochondria are a source of oxidants and mitochondria-related proteins control the activation of the NLRP3 inflammasome. The increased oxidative stress in COPD patients has been linked to mitochondrial dysfunction, which is partly characterised by the impaired translocation of Parkin following activation of the Pink1 mitophagy pathway. Further mitochondrial pathways are likely to be altered in COPD and candidates for further investigation include mitophagy pathways induced by BNIP3 and FUNDC1, and mitochondrial biogenesis pathways.

Although mitochondrial dysfunction seems to be a common feature in COPD patients, the specific dysregulated mitochondrial pathways leading to carcinogenesis remain to be identified. Therefore, future studies should attempt to define the mitochondria-related pathways which contribute to increased oxidative stress and chronic inflammation in COPD and lung cancer and delineate those COPD patients who will go on to develop cancer. Selective blockade of these pathways may provide novel avenues for therapeutic intervention and the use of mitochondria-based antioxidants should be studied as an adjunct in reducing the risk of COPD-associated cancer in animal models. Future clinical trials may then consider the efficacy of mitochondria-targeted antioxidants as a prophylactic agent in reducing the incidence of cancer in those at high risk.

Understanding the mitochondrial dysfunctions in COPD and potentially lung cancer may contribute to the generation of specific biomarkers that would fine-tune the screening strategy. The development of these biomarkers may help to identify COPD patients who will go on to develop lung cancer. The possibility of treating established lung cancers with mitochondria-targeted agents as an adjunct will also have to be considered.

\section{References}

1 Jemal A, Bray F, Center MM, et al. Global cancer statistics. CA Cancer J Clin 2011; 61: 69-90.

2 Molina JR, Yang P, Cassivi SD, et al. Non-small cell lung cancer: epidemiology, risk factors, treatment, and survivorship. Mayo Clin Proc 2008; 83: 584-594.

3 Siegel R, Naishadham D, Jemal A. Cancer statistics, 2012. CA cancer J 2012; 62: 10-29.

4 Lee CK, Brown C, Gralla RJ, et al. Impact of EGFR inhibitor in non-small cell lung cancer on progression-free and overall survival: a meta-analysis. J Natl Cancer Inst 2013; 105: 595-605.

5 Gainor JF, Tan DSW, De Pas T, et al. Progression-free and overall survival in ALK-positive NSCLC patients treated with sequential crizotinib and ceritinib. Clin Cancer Res 2015; 21: 2745-2752.

6 Peters S, Adjei A, Gridelli C, et al. Metastatic non-small-cell lung cancer (NSCLC): ESMO Clinical Practice Guidelines for diagnosis, treatment and follow-up. Ann Oncol 2012; 23: Suppl. 7, vii56-vii64.

7 Ferlay J, Shin HR, Bray F, et al. Estimates of worldwide burden of cancer in 2008: GLOBOCAN 2008. Int $J$ Cancer 2010; 127: 2893-2917.

8 Young RP, Hopkins RJ, Christmas T, et al. COPD prevalence is increased in lung cancer, independent of age, sex and smoking history. Eur Respir J 2009; 34: 380-386.

9 Wilson DO, Weissfeld JL, Balkan A, et al. Association of radiographic emphysema and airflow obstruction with lung cancer. Am J Respir Crit Care Med 2008; 178: 738-744.

10 Tockman MS, Anthonisen NR, Wright EC, et al. Airways obstruction and the risk for lung cancer. Ann Intern Med 1987; 106: 512-518.

11 Vineis P, Airoldi L, Veglia F, et al. Environmental tobacco smoke and risk of respiratory cancer and chronic obstructive pulmonary disease in former smokers and never smokers in the EPIC prospective study. BMJ 2005; 330: 277.

12 Skillrud DM, Offord KP, Miller RD. Higher risk of lung cancer in chronic obstructive pulmonary disease: a prospective, matched, controlled study. Ann Intern Med 1986; 105: 503-507.

13 Speizer FE, Fay ME, Dockery DW, et al. Chronic obstructive pulmonary disease mortality in six US cities. Am Rev Respir Dis 1989; 140: S49-S55.

14 Lange P, Nyboe J, Appleyard M, et al. Ventilatory function and chronic mucus hypersecretion as predictors of death from lung cancer. Am Rev Respir Dis 1990; 141: 613-617.

15 Mannino DM, Aguayo SM, Petty TL, et al. Low lung function and incident lung cancer in the United States: data from the First National Health and Nutrition Examination Survey follow-up. Arch Intern Med 2003; 163: 1475-1480.

16 Calabrò E, Randi G, La Vecchia C, et al. Lung function predicts lung cancer risk in smokers: a tool for targeting screening programmes. Eur Respir J 2010; 35: 146-151. 
De Torres JP, Maron JM, Casanova C, et al. Lung cancer in patients with chronic obstructive pulmonary disease: incidence and predicting factors. Am J Respir Crit Care Med 2011; 184: 913-919.

Kuo CH, Wu CY, Lee KY, et al. Chronic obstructive pulmonary disease in stage I non-small cell lung cancer that underwent anatomic resection: the role of a recurrence promoter. COPD 2014; 11: 407-413.

Gullón JA, Suárez I, Medina A, et al. Role of emphysema and airway obstruction in prognosis of lung cancer. Lung Cancer 2011; 71: 182-185.

Smith BM, Schwartzman K, Kovacina B, et al. Lung cancer histologies associated with emphysema on computed tomography. Lung Cancer 2012; 76: 61-66.

Gao Y, Guan W, Liu Q, et al. Impact of COPD and emphysema on survival of patients with lung cancer: a meta-analysis of observational studies. Respirology 2015; 21: 269-279.

Oelsner EC, Carr JJ, Enright PL, et al. Per cent emphysema is associated with respiratory and lung cancer mortality in the general population: a cohort study. Thorax 2016; 71: 624-632.

Dennis MK, Field AS, Burai R, et al. Correlation of regional emphysema and lung cancer: a lung tissue research consortium based study. J Thorac Oncol 2014; 127: 358-366.

Bishawi M, Moore W, Bilfinger T. Severity of emphysema predicts location of lung cancer and 5-y survival of patients with stage I non-small cell lung cancer. J Surg Res 2013; 184: 1-5.

Nakamura H, Saji H. Worldwide trend of increasing primary adenocarcinoma of the lung. Surg Today 2014; 44: 1004-1012.

Pallis AG, Syrigos KN. Lung cancer in never smokers: disease characteristics and risk factors. Crit Rev Oncol Hematol 2013; 88: 494-503.

Yatabe Y. EGFR mutations and the terminal respiratory unit. Cancer Metastasis Rev 2010; 29: 23-36.

Minuti G, D’Incecco A, Cappuzzo F. Targeted therapy for NSCLC with driver mutations. Expert Opin Biol Ther 2013; 13: 1401-1412.

Gou LY, Niu FY, Wu YL, et al. Differences in driver genes between smoking-related and non-smoking-related lung cancer in the Chinese population. Cancer 2015; 121: Suppl. 17, 3069-3079.

Travis WD, Brambilla E, Burke AP, et al., eds. Chapter 1: Tumours of the lung. In: WHO Classification of Tumours of the Lung, Pleura, Thymus and Heart. Geneva, WHO Press, 2015; pp. 51-55.

Poullis M, McShane J, Shaw M, et al. Smoking status at diagnosis and histology type as determinants of long-term outcomes of lung cancer patients. Eur J Cardiothorac Surg 2013; 43: 919-924.

Sun S, Schiller JH, Gazdar AF. Lung cancer in never smokers - a different disease. Nature 2007; 7: 778-790.

Lim JU, Yeo CD, Rhee CK, et al. Chronic obstructive pulmonary disease-related non-small-cell lung cancer exhibits a low prevalence of EGFR and ALK driver mutations. PLoS One 2015; 10: e0142306.

Saber A, van der Wekken AJ, Kerner GS, et al. Chronic obstructive pulmonary disease is not associated with KRAS mutations in non-small cell lung cancer. PLoS One 2016; 11: e0152317.

Papi A, Casoni G, Caramori G, et al. COPD increases the risk of squamous histological subtype in smokers who develop non-small cell lung carcinoma. Thorax 2004; 59: 679-681.

Li Y, Swensen SJ, Karabekmez LG, et al. Effect of emphysema on lung cancer risk in smokers: a computed tomography-based assessment. Cancer Prev Res 2011; 4: 43-50.

Shin B, Shin S, Chung MJ, et al. Different histological subtypes of peripheral lung cancer based on emphysema distribution in patients with both airflow limitation and CT-determined emphysema. Lung Cancer 2017; 104: $106-110$.

Rowbotham SP, Kim CF. Diverse cells at the origin of lung adenocarcinoma. Proc Natl Acad Sci USA 2014; 111: 4745-4746.

Boelens MC, Gustafson AM, Postma DS, et al. A chronic obstructive pulmonary disease related signature in squamous cell lung cancer. Lung Cancer 2011; 72: 177-183.

Wauters E, Janssens W, Vansteenkiste J, et al. DNA methylation profiling of non-small cell lung cancer reveals a COPD-driven immune-related signature. Thorax 2015; 70: 1113-1122.

Peng H, Yang M, Chen Z, et al. Expression and methylation of mitochondrial transcription factor a in chronic obstructive pulmonary disease patients with lung cancer. PLoS One 2013; 8: e82739.

azuki M, Wada $\mathrm{H}$, Yoshino $\mathrm{M}$, et al. Molecular characterization of chronic obstructive pulmonary disease-related non-small cell lung cancer through aberrant methylation and alterations of EGFR signaling. Ann Surg Oncol 2010; 17: 878-888.

Xiao D, Li F, Pan H, et al. Integrative analysis of genomic sequencing data reveals higher prevalence of LRP1B mutations in lung adenocarcinoma patients with COPD. Sci Rep 2017; 7: 2121.

Bozinovski S, Vlahos R, Anthony D, et al. COPD and squamous cell lung cancer: aberrant inflammation and immunity is the common link. Br J Pharmacol 2015; 173: 635-648.

Rooney C, Sethi T. The epithelial cell and lung cancer: The link between chronic obstructive pulmonary disease and lung cancer. Respiration 2011; 81: 89-104.

Sekine Y, Hata A, Koh E, et al. Lung carcinogenesis from chronic obstructive pulmonary disease: characteristic of lung cancer from COPD and contribution of signal transducers and lung stem cells in the inflammatory microenvironment. Gen Thorac Cardiovasc Surg 2014; 62: 415-421.

Bhattacharyya S, Saha J. Tumour, oxidative stress and host T cell response: cementing the dominance. Scand Immunol 2015; 82: 477-488.

Mantovani A, Allavena P, Sica A, et al. Cancer-related inflammation. Nature 2008; 454: 436-444.

Wiegman $\mathrm{CH}$, Michaeloudes C, Haji G, et al. Oxidative stress-induced mitochondrial dysfunction drives inflammation and airway smooth muscle remodeling in patients with chronic obstructive pulmonary disease. J Allergy Clin Immunol 2015; 136: 769-780.

Pierrou S, Broberg P, O’Donnell RA, et al. Expression of genes involved in oxidative stress responses in airway epithelial cells of smokers with chronic obstructive pulmonary disease. Am J Respir Crit Care Med 2007; 175: 577-586.

51 Yamada K, Asai K, Nagayasu F, et al. Impaired nuclear factor erythroid 2-related factor 2 expression increases apoptosis of airway epithelial cells in patients with chronic obstructive pulmonary disease due to cigarette smoking. BMC Pulm Med 2016; 16: 27.

Marnett LJ. Oxyradicals and DNA damage. Carcinogenesis 2000; 21: 361-370. 
Caramori G, Adcock IM, Casolari P, et al. Unbalanced oxidant-induced DNA damage and repair in COPD: a link towards lung cancer. Thorax 2011; 66: 521-527.

Pastukh VM, Zhang L, Vruchko M, et al. Oxidative DNA damage in lung tissue from patients with COPD is clustered in functionally significant sequences. Int J COPD 2011; 6: 209-217.

Lantuejoul S, Raynaud C, Salameire D, et al. Telomere maintenance and DNA damage responses during lung carcinogenesis. Clin Cancer Res 2010; 16: 2979-2988.

Park IW, Wistuba II, Maitra A, et al. Multiple clonal abnormalities in the bronchial epithelium of patients with lung cancer. J Natl Cancer Inst 1999; 91: 1863-1868.

Pastor MD, Nogal A, Molina-Pinelo S, et al. Identification of oxidative stress related proteins as biomarkers for lung cancer and chronic obstructive pulmonary disease in bronchoalveolar lavage. Int J Mol Sci 2013; 14: 3440-3455.

Barnes PJ. Senescence in COPD and its comorbidities. Annu Rev Physiol 2017; 79: 517-539.

Correia-Melo C, Birch J, Passos JF. Powering senescence: the ugly side of mitochondria. Cell Cycle 2016; 15: 2541-2542.

Passos JF, Saretzki G, von Zglinicki T. DNA damage in telomeres and mitochondria during cellular senescence: is there a connection? Nucleic Acids Res 2007; 35: 7505-7513.

Passos JF, Saretzki G, Ahmed S, et al. Mitochondrial dysfunction accounts for the stochastic heterogeneity in telomere-dependent senescence. PLoS Biol 2007; 5: e110.

Wiley CD, Velarde MC, Lecot $\mathrm{P}$, et al. Mitochondrial dysfunction induces senescence with a distinct secretory phenotype. Cell Metab 2016; 23: 303-314.

Campisi J, Andersen JK, Kapahi P, et al. Cellular senescence: a link between cancer and age-related diseases? Semin Cancer Biol 2011; 21: 354-359.

Baker DJ, Alimirah F, van Deursen JM, et al. Oncogenic senescence: a multi-functional perspective. Oncotarget 2017; 8: 27661-27672.

Bhat TA, Panzica L, Kalathil SG, et al. Immune dysfunction in patients with chronic obstructive pulmonary disease. Ann Am Thorac Soc 2015; 12: Suppl. 2, S169-S175.

McKendry RT, Spalluto CM, Burke H, et al. Dysregulation of antiviral function of CD8+ T cells in the chronic obstructive pulmonary disease lung. Role of the PD-1-PD-L1 axis. Am J Respir Crit Care Med 2016; 193: 642-651.

Grivennikov SI, Karin M. Inflammation and oncogenesis: a vicious connection. Curr Opin Genet Dev 2010; 20: 65-71.

Siemes C, Visser LE, Coebergh JW, et al. C-reactive protein levels, variation in the C-reactive protein gene, and cancer risk: the Rotterdam Study. J Clin Oncol 2006; 24: 5216-5222.

Parimon T, Chien JW, Bryson CL, et al. Inhaled corticosteroids and risk of lung cancer among patients with chronic obstructive pulmonary disease. Am J Respir Crit Care Med 2007; 175: 712-719.

Yun J, Finkel T. Mitohormesis. Cell Metab 2014; 19: 757-766.

Trends Mol Med 2015; 21: 193-201.

Martinon F, Burns K, Tschopp J. The inflammasome: a molecular platform triggering activation of inflammatory caspases and processing of proIL-beta. Mol Cell 2002; 10: 417-426.

Davis BK, Wen H, Ting JP. The inflammasome NLRs in immunity, inflammation, and associated diseases. Annu Rev Immunol 2011; 29: 707-735.

Warburg O. On respiratory impairment in cancer cells. Science 1956; 124: 269-270.

Zong WX, Rabinowitz JD, White E. Mitochondria and cancer. Mol Cell 2016; 61: 667-676.

Wang L, Chen ZJ, Zhang YK, et al. The role of mitochondrial tRNA mutations in lung cancer. Int J Clin Exp Med 2015; 8: 13341-13346.

Wang Z, Choi S, Lee J, et al. Mitochondrial variations in non-small cell lung cancer (NSCLC) survival. Cancer Inform 2015; 14: Suppl. 1, 1-9.

Akgul EO, Kurt B, Gulcan Kurt Y, et al. MtDNA depletions and deletions may also be important in pathogenesis of lung cancer. Respir Med 2013; 107: 1814.

Qi Y, Wei Y, Wang Q, et al. Heteroplasmy of mutant mitochondrial DNA A10398G and analysis of its prognostic value in non-small cell lung cancer. Oncol Lett 2016; 12: 3081-3088.

Yang Ai SS, Hsu K, Herbert C, et al. Mitochondrial DNA mutations in exhaled breath condensate of patients with lung cancer. Respir Med 2013; 107: 911-918.

Aravamudan B, Kiel A, Freeman M, et al. Cigarette smoke-induced mitochondrial fragmentation and dysfunction in human airway smooth muscle. Am J Physiol Lung Cell Mol Physiol 2014; 306: L840-L854.

Ballinger SW, Bouder TG, Davis GS, et al. Mitochondrial genome damage associated with cigarette smoking. Cancer Res 1996; 56: 5692-5697.

Hoffmann RF, Zarrintan S, Brandenburg SM, et al. Prolonged cigarette smoke exposure alters mitochondria structure and function in airway epithelial cells. Respir Res 2013; 14: 97. adducts to mitochondrial DNA in rat organs. Cancer Res 1996; 56: 1642-1647.

Stepanov I, Hecht SS. Mitochondrial DNA adducts in the lung and liver of F344 rats chronically treated with 4-(methylnitrosamino)-1-(3-pyridyl)-1-butanone and (S)-4-(methylnitrosamino)-1-(3-pyridyl)-1-butanol. Chem Res Toxicol 2009; 22: 406-414.

Bodhicharla R, Ryde IT, Pradad GL, et al. The tobacco-specific nitrosamine 4-(methylnitrosamino)-1(3-pyridyl)-1-butanone (NNK) induces mitochondrial and nuclear DNA damage in Caenorhabditis elegans. Environ Mol Mutagen 2014; 55: 43-50.

Ballweg K, Mutze K, Konigstoff $\mathrm{M}$, et al. Cigarette smoke extract affects mitochondrial function in alveolar epithelial cells. Am J Physiol Lung Cell Med Physiol 2014; 307: L895-L907.

Rehman J, Zhang HJ, Toth PT, et al. Inhibition of mitochondrial fission prevents cell cycle progression in lung cancer. FASEB J 2012; 26: 2175-2186.

Lou Y, Li R, Liu J, et al. Mitofusin-2 over-expresses and leads to dysregulation of cell cycle and cell invasion in lung adenocarcinoma. Med Oncol 2015; 32. 
Polosukhin VV, Cates JM, Lawson WE, et al. Hypoxia-inducible factor-1 signaling promotes goblet cell hyperplasia in airway epithelium. J Pathol 2011; 224: 203-211.

Ping W, Jiang WY, Chen WS, et al. Expression and significance of hypoxia inducible factor-1 $\alpha$ and lysyl oxidase in non-small cell lung cancer. Asian Pac J Cancer Prev 2013; 14: 3613-3618.

Hung JJ, Yang MH, Hsu HS, et al. Prognostic significance of hypoxia-inducible factor-1 $\alpha$, TWIST1 and Snail expression in resectable non-small cell lung cancer. Thorax 2009; 64: 1082-1089.

Kao CC, Hsu JW, Bandi V, et al. Glucose and pyruvate metabolism in severe chronic obstructive pulmonary disease. J Appl Physiol 2012; 112: 42-47.

Schönenberger MJ, Kovacs WJ. Hypoxia signaling pathways: modulators of oxygen-related organelles. Front Cell Dev Biol 2015; 3: 42.

Zhang J, Ney PA. Role of BNIP3 and NIX in cell death, autophagy, and mitophagy. Cell Death Differ 2009; 16 939-946.

Novak I. Mitophagy: a complex mechanism of mitochondrial removal. Antioxid Redox Signal 2012; 17: 794-802. Feng D, Liu L, Zhu Y, et al. Molecular signaling toward mitophagy and its physiological significance. Exp Cell Res 2013; 319: 1697-1705.

Liu L, Feng D, Chen G, et al. Mitochondrial outer-membrane protein FUNDC1 mediates hypoxia-induced mitophagy in mammalian cells. Nat Cell Biol 2012; 14: 177-185.

Hanna RA, Quinsay MN, Orogo AM, et al. Microtubule-associated protein 1 light chain 3 (LC3) interacts with Bnip3 protein to selectively remove endoplasmic reticulum and mitochondria via autophagy. J Biol Chem 2012; 287: 19094-19104.

Schwarten M, Mohrlüder J, Ma P, et al. Nix directly binds to GABARAP: a possible crosstalk between apoptosis and autophagy. Autophagy 2009; 5: 690-698.

Narendra DP, Jin SM, Tanaka A, et al. PINK1 is selectively stabilized on impaired mitochondria to activate Parkin. PLoS Biol 2010; 8: e1000298.

Narendra D, Walker JE, Youle R. Mitochondrial quality control mediated by PINK1 and Parkin: links to parkinsonism. Cold Spring Harb Perspect Biol 2012; 4: a011338.

Murphy MP. How mitochondria produce reactive oxygen species. Biochem J 2009; 417: 1-13.

Saitoh T, Fujita N, Jang MH, et al. Loss of the autophagy protein Atg16L1 enhances endotoxin-induced IL-1beta production. Nature 2008; 456: 264-268.

Bensaad K, Cheung EC, Vousden KH. Modulation of intracellular ROS levels by TIGAR controls autophagy. EMBO J 2009; 28: 3015-3026.

Nakahira K, Haspel JA, Rathinam VAK, et al. Autophagy proteins regulate innate immune responses by inhibiting the release of mitochondrial DNA mediated by the NALP3 inflammasome. Nat Immunol 2011; 12: $222-230$.

Zhou R, Yazdi AS, Menu P, et al. A role for mitochondria in NLRP3 inflammasome activation. Nature 2010; 469: $221-225$

Morselli E, Galluzzi L, Kepp O, et al. Oncosuppressive functions of autophagy. Antioxid Redox Signal 2011; 14: 2251-2269.

Mizumura K, Cloonan SM, Nakahira K, et al. Mitophagy-dependent necroptosis contributes to the pathogenesis of COPD. J Clin Invest 2014; 124: 3987-4003.

Ito S, Araya J, Kurita Y, et al. PARK2-mediated mitophagy is involved in regulation of HBEC senescence in COPD pathogenesis. Autophagy 2015; 11: 547-559.

Ahmad T, Sundar IK, Lerner CA, et al. Impaired mitophagy leads to cigarette smoke stress-induced cellular senescence: implications for chronic obstructive pulmonary disease. FASEB J 2015; 29: 2912-2929.

Berthier A, Navarro S, Jimrnez-Sainz J, et al. PINK1 displays tissue-specific subcellular location and regulates apoptosis and cell growth in breast cancer cells. Hum Pathol 2011; 42: 75-87.

D'Amico AG, Maugeri G, Magro G, et al. Expression pattern of parkin isoforms in lung adenocarcinomas. Tumour Biol 2015; 36: 5133-5141.

Lommatzsch M, Cicko S, Müller T, et al. Extracellular adenosine triphosphate and chronic obstructive pulmonary disease. Am J Respir Crit Care Med 2010; 181: 928-934.

Brusselle GG, Provoost S, Bracke KR, et al. Inflammasomes in respiratory disease: from bench to bedside. Chest 2014; 145: 1121-1133.

Massaro GD, Gail DB, Massaro D. Lung oxygen consumption and mitochondria of alveolar epithelial and endothelial cells. J Appl Physiol 1975; 38: 588-592.

pulmonary disease. I Innate Immun 2016; 8: 121-128

Kong H, Wang Y, Zeng X, et al. Differential expression of inflammasomes in lung cancer cell lines and tissues. Tumour Biol 2015; 36: 7501-7513.

Wang Y, Wang Y, Kong H, et al. Activation of NLRP3 inflammasome enhances the proliferation and migration of A549 lung cancer cells. Oncol Rep 2016; 35: 2053-2064.

Smith RAJ, Murphy MP. Animal and human studies with the mitochondria-targeted antioxidant MitoQ. Ann N Y Acad Sci 2010; 1201: 96-103.

Szeto HH. Mitochondria-targeted peptide antioxidants: novel neuroprotective agents. AAPS J 2006; 8: E521-E531.

Moreno P, Lara-Chica M, Soler-Torronteras R, et al. The expression of the ubiquitin ligase SIAH2 (Seven In Absentia Homolog 2) is increased in human lung cancer. PLoS One 2015; 10: e0143376.

Cimino Y, Costes A, Damotte D, et al. FADD protein release mirrors the development and aggressiveness of human non-small cell lung cancer. Br J Cancer 2012; 106: 1989-1996.

Meuwissen R, Berns A. Mouse models for human lung cancer. Genes Dev 2005; 19: 643-664.

Guerra C, Mijimolle N, Dhawahir A, et al. Tumor induction by an endogenous K-ras oncogene is highly dependent on cellular context. Cancer Cell 2003; 4: 111-120.

Jackson EL, Willis N, Mercer K, et al. Analysis of lung tumor initiation and progression using conditional expression of oncogenic K-ras. Genes Dev 2001; 15: 3243-3248. 
127 Song L, Smith MA, Doshi P, et al. Antitumor efficacy of the anti-interleukin-6 (IL-6) antibody siltuximab in mouse xenograft models of lung cancer. J Thorac Oncol 2014; 9: 974-982.

128 Malkinson AM, Nesbitt MN, Skamene E. Susceptibility to urethan-induced pulmonary adenomas between A/J and C57BL/6J mice: use of AXB and BXA recombinant inbred lines indicating a three-locus genetic model. J Natl Cancer Inst 1985; 75: 971-974.

129 Shimkin MB, Stoner GD. Lung tumors in mice: application to carcinogenesis bioassay. Adv Cancer Res 1975; 21: $1-58$. 